\title{
Anosognosia predicts default mode network hypometabolism and clinical progression to dementia
}

Joseph Therriault, BA, Kok Pin Ng, MRCP, Tharick A. Pascoal, MD, Sulantha Mathotaarachchi, MSc, Min Su Kang, BSc, Hanne Struyfs, MSc, Monica Shin, MSc, Andrea L. Benedet, MSc, Ishan C. Walpola, MSc, Vasavan Nair, MD, Serge Gauthier, MD, and Pedro Rosa-Neto, MD, PhD, For the Alzheimer's Disease Neuroimaging Initiative

Neurology ${ }^{\circledR}$ 2018;90:e932-e939. doi:10.1212/WNL.0000000000005120

\section{Abstract \\ Objective}

To identify the pathophysiologic mechanisms and clinical significance of anosognosia for cognitive decline in mild cognitive impairment.

\section{Methods}

We stratified 468 patients with amnestic mild cognitive impairment into intact and impaired awareness groups, determined by the discrepancy between the patient and the informant score on the Everyday Cognition questionnaire. Voxel-based linear regression models evaluated the associations between self-awareness status and baseline $\beta$-amyloid load, measured by $\left[{ }^{18} \mathrm{~F}\right]$ florbetapir, and the relationships between awareness status and regional brain glucose metabolism measured by $\left[{ }^{18} \mathrm{~F}\right]$ fluorodeoxyglucose at baseline and at 24-month follow-up. Multivariate logistic regression tested the association of awareness status with conversion from amnestic mild cognitive impairment to dementia.

\section{Results}

We found that participants with impaired awareness had lower $\left[{ }^{18} \mathrm{~F}\right]$ fluorodeoxyglucose uptake and increased $\left[{ }^{18} \mathrm{~F}\right]$ florbetapir uptake in the posterior cingulate cortex at baseline. In addition, impaired awareness in mild cognitive impairment predicted $\left[{ }^{18} \mathrm{~F}\right]$ fluorodeoxyglucose hypometabolism in the posterior cingulate cortex, left basal forebrain, bilateral medial temporal lobes, and right lateral temporal lobe over 24 months. Furthermore, participants with impaired awareness had a nearly 3 -fold increase in likelihood of conversion to dementia within a 2-year time frame.

\section{Conclusions}

Our results suggest that anosognosia is linked to Alzheimer disease pathophysiology in vulnerable structures, and predicts subsequent hypometabolism in the default mode network, accompanied by an increased risk of progression to dementia. This highlights the importance of assessing awareness of cognitive decline in the clinical evaluation and management of individuals with amnestic mild cognitive impairment.

\author{
Correspondence \\ Dr. Rosa-Neto \\ pedro.rosa@mcgill.ca
}




\section{Glossary}

$\mathbf{A} \beta=\beta$-amyloid $\mathbf{A D}=$ Alzheimer disease; ADAS-Cog = Alzheimer's Disease Assessment Scale-cognitive subscale; $\mathbf{A D N I}=$ Alzheimer's Disease Neuroimaging Initiative; ANCOVA = analysis of covariance; CI = confidence interval; DMN = default mode network; ECog = everyday cognition; $\mathbf{M C I}=$ mild cognitive impairment; MMSE = Mini-Mental State Examination; OR = odds ratio; $\mathbf{p}$-tau = phosphorylated tau; $\mathbf{P C C}=$ posterior cingulate cortex; RAVLT-D = Rey Auditory Verbal Learning Task-delayed recall; RAVLT-IM = Rey Auditory Verbal Learning Task-immediate recall memory; SUVR = standardized uptake value ratio; TMT-A = Trail-Making Test part A; TMT-B = Trail-Making Test part B.

Anosognosia, a syndrome characterized by a lack of awareness of one's illness, is commonly observed in Alzheimer disease $(\mathrm{AD}){ }^{1}$ Patients with $\mathrm{AD}$ with anosognosia are unaware of their cognitive deficits and the difficulties they face when performing activities of daily living. Anosognosia is also correlated with dementia severity in $\mathrm{AD}^{2}$ Hence, anosognosia constitutes an important clinical characteristic of patients with $\mathrm{AD}$.

The relationship between anosognosia and $\mathrm{AD}$ pathophysiology in the predementia phase of $\mathrm{AD}$ is poorly understood. Because anosognosia occurs frequently in $\mathrm{AD}$, further studies focusing on the associations of anosognosia in mild cognitive impairment (MCI) and $\mathrm{AD}$ pathophysiology are of paramount importance, given that anosognosia in MCI may represent an important clinical syndrome that signals disease progression. ${ }^{3}$

The present study aims to characterize the associations of anosognosia and $\mathrm{AD}$ biomarkers based on the recently described amyloid/tau/neurodegeneration construct ${ }^{4}$ in $\mathrm{MCI}$, using $\left[{ }^{18} \mathrm{~F}\right]$ florbetapir, CSF phosphorylated tau (p-tau), and $\left[{ }^{18} \mathrm{~F}\right] \mathrm{FDG}$, respectively. In addition, we examine the predictive effect of anosognosia on disease progression and changes in brain glucose metabolism at a 24-month follow-up. Because anosognosia occurs frequently in the symptomatic phase of $\mathrm{AD}$, we also hypothesized that anosognosia is related to an increase in neuropathologic features of $\mathrm{AD}$ at baseline, and correspondingly to higher rates of diagnostic conversion at 24-month follow-up. The finding of a significant relationship between anosognosia, $\mathrm{AD}$ biomarkers, and disease progression would support a framework where assessments of illness awareness constitute an important role in the clinical management of MCI.

\section{Methods}

\section{Participants}

Data used in the preparation of this article were obtained from the Alzheimer's Disease Neuroimaging Initiative (ADNI) database (adni.loni.usc.edu). The ADNI was launched in 2003 as a public-private partnership led by principal investigator Michael W. Weiner, MD. The primary goal of ADNI has been to test whether serial MRI, PET, other biological markers, and clinical and neuropsychological assessment can be combined to measure the progression of $\mathrm{MCI}$ and early $\mathrm{AD}$.
In this study, we selected participants meeting criteria for amnestic MCI who have both their baseline everyday cognition $(\mathrm{ECog})^{5}$ as well as their study partner's ECog scores. Participants with MCI were defined as having (1) a subjective memory concern as reported by the participant, study partner, or clinician; (2) abnormal memory function documented by scoring within education-adjusted ranges; (3) Mini-Mental State Examination (MMSE) score between 24 and 30; (4) Clinical Dementia Rating (CDR) 0.5 with a memory box score of at least 0.5 ; and (5) general cognition and functional performance sufficiently preserved such that a diagnosis of $\mathrm{AD}$ cannot be made by the site physician at the time of the screening visit. The ADNI inclusion and exclusion criteria can be accessed at adni-info.org (accessed January 2017).

\section{Standard protocol approvals, registrations, and patient consents}

The ADNI study was approved by the institutional review boards of each participating institution. Informed written consent was obtained from all participants enrolled in this study.

\section{Determining intact and impaired self- awareness}

We defined intact and impaired self-awareness of cognitive deficits based on the difference between patient and informant global ratings on the ECog questionnaire. Participants with anosognosia are described as those with impaired self-awareness. The ECog questionnaire measures global as well as domain-specific cognitive functions, such as episodic memory and planning, by asking participants to compare their cognitive abilities currently and 10 years earlier. A study partner, such as a spouse or caregiver, who spent a minimum of 10 hours per week with the participant, also completed the ECog questionnaire according to the participant's cognitive abilities. ECog is a useful measure for levels of awareness along the $\mathrm{AD}$ spectrum because understanding awareness of deficits in functioning requires the individual to consider performance on everyday activities, where deficits can be observed in MCI. The information provided by caregivers has repeatedly been shown to be highly accurate. ${ }^{6-8}$

The cutoff for the threshold of impaired self-awareness was calculated using the best operational point on a receiver operating characteristic curve contrasting healthy controls $(\mathrm{n}=$ $290)$ and patients with $\mathrm{AD}(\mathrm{n}=150)$ from the ADNI cohort. A cutoff of 0.5 , determined by the threshold that optimized both 
sensitivity and specificity, was subsequently used to stratify the participants with MCI into intact and impaired self-awareness groups. See figure 1 for a graphical representation of the determination of intact and impaired awareness groups.

\section{Cognitive assessments}

Global cognitive abilities were evaluated using the MMSE and the Alzheimer's Disease Assessment Scale-cognitive subscale (ADAS-Cog). Language abilities were evaluated used the Animals section of the Category Fluency task. The TrailMaking Test part A (TMT-A) was employed to assess psychomotor speed processing, while part B (TMT-B) was used to assess mental flexibility. Finally, the Rey Auditory Verbal Learning Task (RAVLT-IM) was used to assess immediate recall memory and the 30-minute delayed recall (RAVLT-D) was used to assess delayed recall.

\section{CSF analyses}

CSF $\beta$-amyloid $(A \beta)$ and $p$-tau concentrations were measured with the Luminex (Austin, TX) multiplex platform and Innogenetics (Ghent, Belgium) INNO-BIAALZBio3 immunoassay reagents. CSF biomarker data were obtained from the UPENNBIOMK5-8.csv files from ADNI. In the present study, we employed the most recent measure of CSF A $\beta_{1-42}$, tau, and p-tau. Complete details of the methods for acquisition and measurement of CSF in ADNI can be found at adni. info.org (accessed February 2017).

\section{Neuroimaging methods}

A schematic representation of the neuroimaging methods employed is presented in figure e-1 (links.lww.com/WNL/ A235). T1-weighted MRI were acquired following the ADNI acquisition protocols. For complete details of the ADNI neuroimaging data acquisition protocol, see adni.loni.usc. edu/methods/(accessed October 2017). Standardized uptake value ratio (SUVR) maps were obtained for $\left[{ }^{18} \mathrm{~F}\right]$ florbetapir using cerebellar gray matter as the reference region and normalizing for cortical white matter, and $\left[{ }^{18} \mathrm{~F}\right] \mathrm{FDG}$ using the pons as the reference region. A global $\left[{ }^{18} \mathrm{~F}\right]$ florbetapir and $\left[{ }^{18} \mathrm{~F}\right]$ FDG SUVR value for each participant was estimated as the mean SUVR of a brain mask comprising the precuneus, prefrontal, orbitofrontal, parietal, temporal, anterior, and posterior cingulate cortices. PET images were processed using an established pipeline. ${ }^{9}$

\section{Statistical analyses}

Statistical analyses were performed using the R Statistical Software Package version 3.1.2 (r-project.org/; accessed February 2017). Analyses of neuroimaging data were conducted using the RMINC library, a biomedical imaging package that permits Medical Image NetCDF (MINC) files to be analyzed in the $\mathrm{R}$ environment.

Baseline demographics were assessed using multiple $t$ tests and $\chi^{2}$ tests. To characterize intact and impaired awareness groups on $\mathrm{AD}$ biomarkers and neurocognitive functioning, we performed an analysis of covariance (ANCOVA). To account for disease severity, we repeated each ANCOVA model of CSF pathology employing ADAS-Cog scores as an additional covariate.

A voxel-based regression model was used to compare patterns of brain metabolism measured by $\left[{ }^{18} \mathrm{~F}\right]$ FDG PET between groups. Furthermore, we employed a voxel-based regression model to compare patterns of regional amyloid deposition as determined by $\left[{ }^{18} \mathrm{~F}\right]$ florbetapir PET. Of the 124 participants

Figure 1 Determination of intact and impaired awareness groups

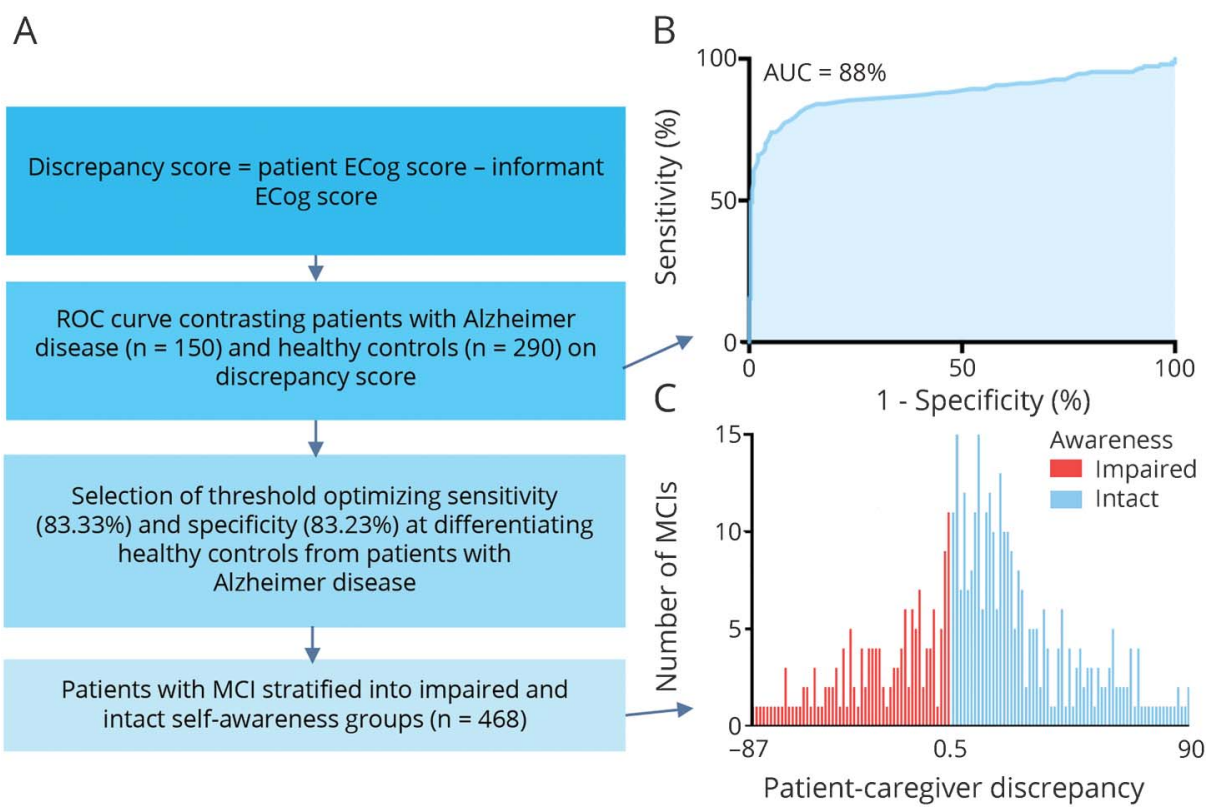

(A) Steps for determining intact and impaired awareness groups. The threshold for the discrepancy score that optimized sensitivity and specificity at differentiating between patients with Alzheimer disease (AD) and healthy controls is 0.5. (B) Receiver operating characteristic (ROC) curve displays sensitivity and specificity at distinguishing between patients with $A D$ and healthy controls. (C) Distribution of participants with mild cognitive impairment $(\mathrm{MCl})$ according to patientcaregiver discrepancy on everyday cognition (ECog). Patient-caregiver discrepancy scores lower than 0.5 indicate impaired awareness (red) and scores above 0.5 indicate intact awareness (blue). AUC = area under the curve. 
Table Demographics and key characteristics of the mild cognitive impairment population

\begin{tabular}{llll}
\hline Characteristics & $\begin{array}{l}\text { Intact self- } \\
\text { awareness }\end{array}$ & $\begin{array}{l}\text { Impaired self- } \\
\text { awareness }\end{array}$ & $\boldsymbol{p}$ Value \\
\hline $\mathbf{n}$ & 293 & 175 & - \\
\hline Age, y, mean (SD) & $70.72(7.74)$ & $72.24(7.04)$ & 0.035 \\
\hline Female, $\mathbf{n}(\%)$ & $148(69.48)$ & $65(30.52)$ & 0.0054 \\
\hline APOE \&4, $\mathbf{n}$ (\%) & $129(44.64)$ & $94(53.71)$ & 0.0685 \\
\hline $\begin{array}{l}\text { Education, } \mathbf{y}, \\
\text { mean (SD) }\end{array}$ & $16.17(2.64)$ & $16.1(2.7)$ & 0.7723 \\
\hline
\end{tabular}

$p$ Values indicate the values assessed with independent samples $t$ tests for each variable except sex, $A P O E \& 4$, and diagnostics at follow-up, where contingency $\chi^{2}$ tests were performed.

with amnestic MCI with caregiver and informant E-Cog data with $\left[{ }^{18} \mathrm{~F}\right]$ FDG PET assessments at both baseline and 24-month follow-up, we employed simple linear regression models to evaluate the effect of awareness group on \% change in regional brain metabolism as assessed by $\left[{ }^{18} \mathrm{~F}\right] \mathrm{FDG}$ PET. Age, sex, years of education, and $A P O E \varepsilon 4$ status were employed as covariates in all voxel-based regression analyses. Because participants with $\mathrm{MCI}$ in the anosognosia group had higher ADAS-Cog scores, we repeated each voxel-based regression model employing ADAS-Cog scores as an additional covariate. All voxel-based regression analyses were corrected for multiple comparisons using random field theory ${ }^{10}$ at $p<0.001$.

To determine if patterns of brain metabolism were abnormal, we subsequently computed $z$ scores for the mean SUVR from the structures with lower $\left[{ }^{18} \mathrm{~F}\right] \mathrm{FDG}$ uptake in individuals with MCI with impaired awareness at baseline and at follow-up. A structure was defined as hypometabolic if the mean $\left[{ }^{18} \mathrm{~F}\right] \mathrm{FDG}$ SUVR in the anosognosia group was significantly lower (95\% confidence interval $[\mathrm{CI}])$ than that of the healthy individuals. Defining abnormality based on 95\% CIs from the healthy population is a commonly employed approach. ${ }^{11,12}$

To evaluate whether anosognosia is an important risk factor for progression from amnestic $\mathrm{MCI}$ to $\mathrm{AD}$, we performed a logistic regression analysis including other known factors related to diagnostic conversion including age, sex, years of education, and $A P O E \varepsilon 4$ status as covariates. We also repeated the logistic regression analysis employing ADAS-Cog scores as an additional covariate to correct for cognitive impairment. Odds ratios (ORs) with respective 95\% CIs were calculated.

\section{Results}

Of 468 participants with amnestic MCI, 175 had impaired self-awareness and 293 had intact self-awareness. Baseline demographics are summarized in the table.

The impaired awareness group demonstrated lower scores on the ADAS-Cog. Furthermore, participants with MCI in the impaired awareness group also demonstrated a larger deficiency on both the RAVLT-IM and RAVLT-D. No statistically significant differences were observed for MMSE, Category Fluency, TMT-A, or TMT-B (figure e-2, links.lww. com/WNL/A235).

ANCOVAs revealed that compared with individuals with MCI with intact self-awareness, individuals with MCI with impaired self-awareness had higher levels of CSF p-tau, CSF total tau, and global $\left[{ }^{18} \mathrm{~F}\right]$ florbetapir uptake. They also demonstrated lower CSF A $\beta$ (figure 2). Furthermore, significant correlations between patient-caregiver discrepancy on the ECog questionnaire and CSF A $\beta(r=0.14, p=0.0045)$, global $\left[{ }^{18} \mathrm{~F}\right]$ florbetapir uptake $(r=-0.15, p=0.0017), \mathrm{CSF}$ p-tau $(r=$ $-0.19, p<0.0001)$, and CSF total tau $(r=-0.20, p<0.0001)$

Figure 2 Anosognosia in mild cognitive impairment is a clinical manifestation of Alzheimer disease (AD) pathophysiology

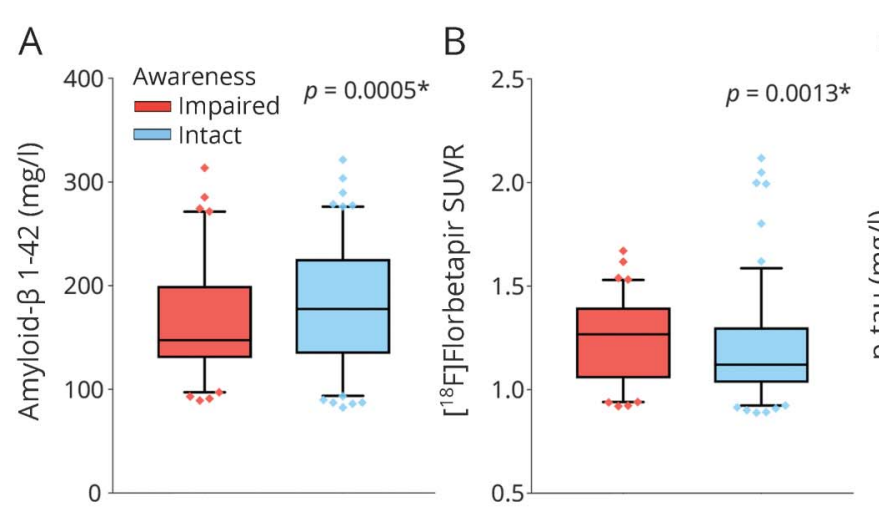

C

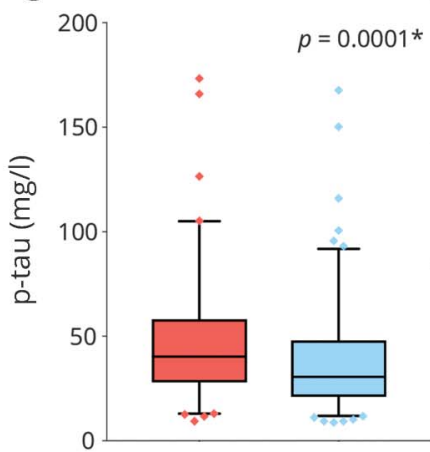

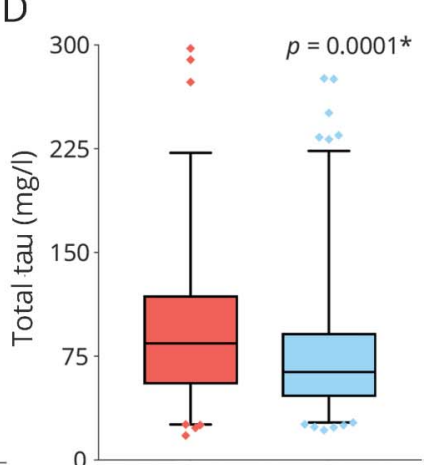

AD biomarker profiles of intact and impaired awareness groups, corrected for age, sex, years of education, and $A P O E \varepsilon 4$ status. Bars in the box and whisker plots display the 5th and 95th percentiles and the horizontal line shows the median. Bonferroni-corrected $p$ values are displayed for each biomarker. For $\beta$-amyloid $(A \beta)_{1-42}$, lower scores indicate lower $A \beta$ concentrations in CSF, thus indicating higher concentrations of brain $A \beta$. When employing Alzheimer's Disease Assessment Scale-cognitive subscale scores as an additional covariate, only total tau remained significant $(p=0.03)$. $p$-tau $=$ phosphorylated tau; SUVR = standardized uptake value ratio. 
were observed. Because 0.5 was the threshold for determining intact vs impaired awareness (with impaired awareness participants having scores below 0.5 ), a negative correlation between $\mathrm{AD}$ biomarkers and patient-caregiver discrepancy is expected.

A voxel-based regression model demonstrated that individuals with amnestic MCI in the impaired self-awareness group had higher $\left[{ }^{18} \mathrm{~F}\right]$ florbetapir uptake in the posterior cingulate cortex (PCC) and right lateral temporal and parietal cortices than individuals with intact self-awareness (figure 3A). Results of a voxel-based regression model revealed that individuals with MCI with impaired awareness had decreased metabolism in the PCC and precuneus (figure 3B). Voxel-based regression model demonstrated that impaired self-awareness in MCI predicts lower $\left[{ }^{18} \mathrm{~F}\right] \mathrm{FDG}$ uptake at 24-month follow-up in the PCC, bilateral medial temporal lobes, right lateral temporal lobe, and basal forebrain (figure 4).

Figure 3 Regional differences in brain metabolism and amyloid deposition
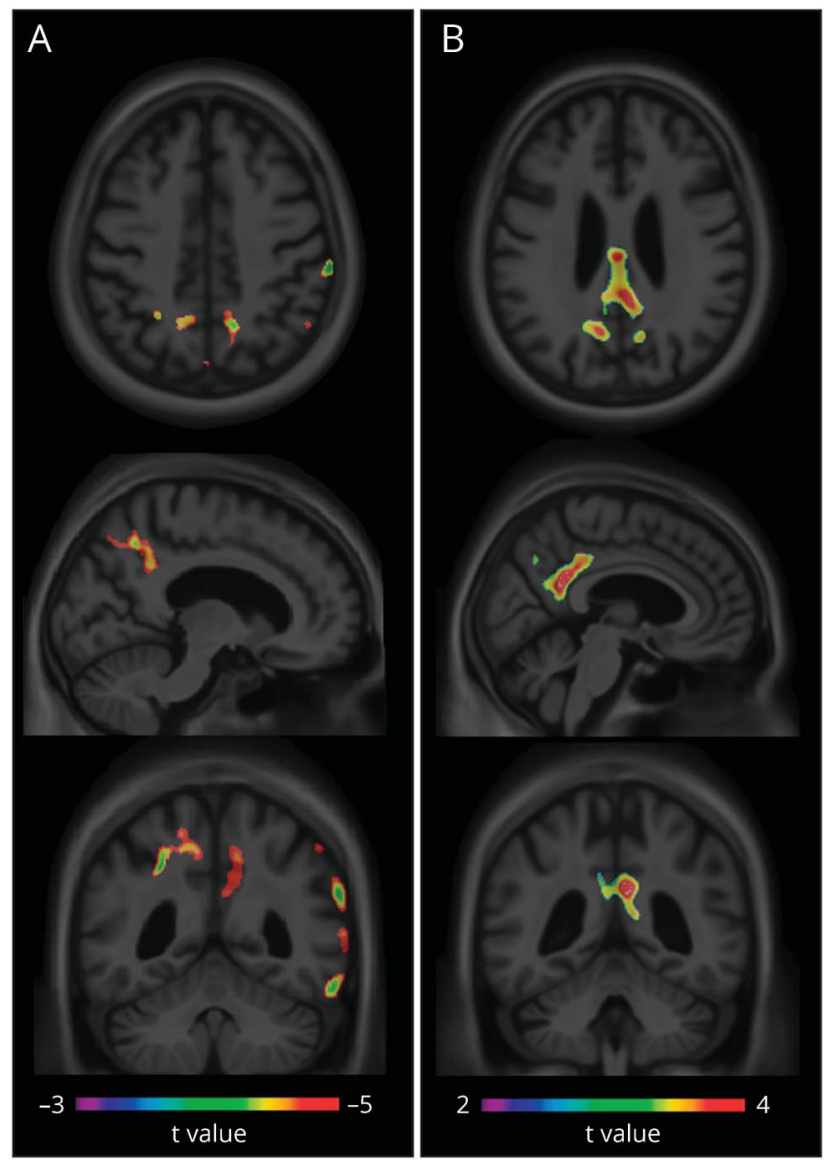

$T$ statistical parametric map corrected for multiple comparisons using random field theory at $p<0.001$, overlaid on the Montreal Neurological Institute 152 reference template. (A) The impaired awareness group had greater $\left[{ }^{18} \mathrm{~F}\right]$ florbetapir binding in the precuneus, posterior cingulate cortex, right lateral parietal, and lateral temporal cortical regions. (B) The impaired awareness group showed decreased $\left[{ }^{18} \mathrm{~F}\right] \mathrm{FDG}$ binding in the posterior cingulate cortex. Both these results remained significant when employing Alzheimer's Disease Assessment Scale-cognitive subscale scores as a covariate.
Figure 4 Anosognosia predicts hypometabolism in neural structures vulnerable to Alzheimer disease

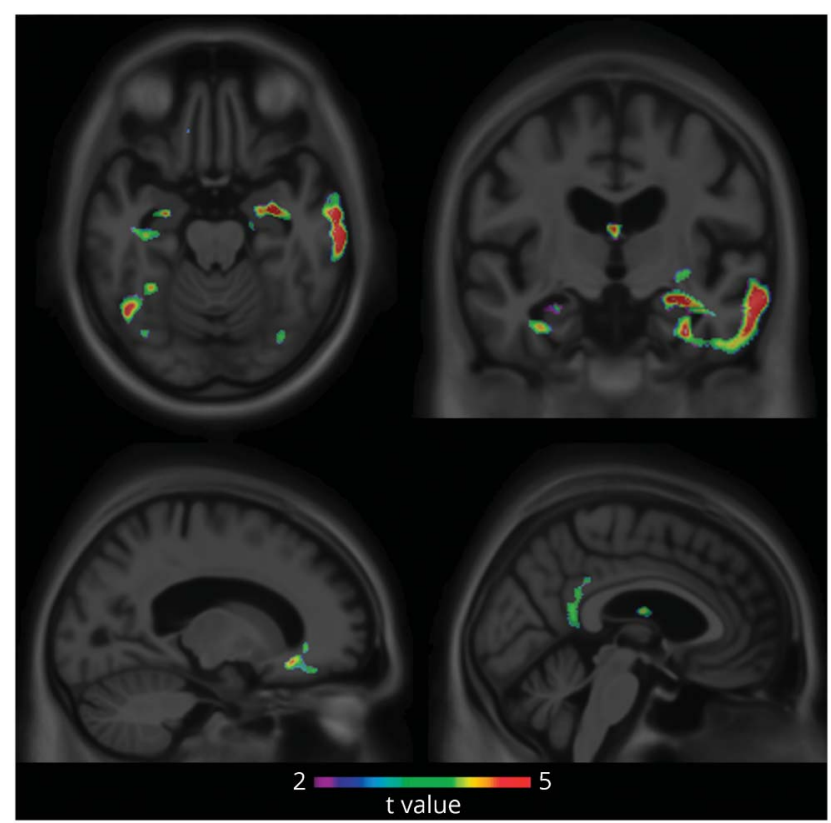

$T$ statistical parametric map corrected for multiple comparisons using random field theory at $p<0.001$, overlaid on the Montreal Neurological Institute 152 reference template. Patients with mild cognitive impairment in the impaired awareness group showed greater decline in $\left[{ }^{18} \mathrm{~F}\right] \mathrm{FDG}$ uptake in the posterior cingulate cortex/precuneus, basal forebrain, and medial temporal lobes at 24-month follow-up compared to patients in the intact awareness group. When employing Alzheimer's Disease Assessment Scale-cognitive subscale scores as a covariate, decline in $\left[{ }^{18} \mathrm{~F}\right] \mathrm{FDG}$ uptake remained significant in the bilateral medial temporal lobes and the basal forebrain.

A total of $28.15 \%$ of patients with amnestic MCI in the anosognosia group converted to $\mathrm{AD}$ dementia, while only $12 \%$ in the intact awareness group converted. A multivariate logistic regression analysis demonstrated that impaired awareness of illness predicts diagnostic conversion over 2 years (OR 2.86, 95\% CI 1.6-5.25). Notably, the only other factor more closely associated with diagnostic conversion at 24-month follow-up was APOE $\varepsilon 4$ status (OR 3.42, 95\% CI 1.88-6.39). Anosognosia remained a significant predictor of progression to dementia (OR 2.83, 95\% CI 1.44-5.7) when ADAS-Cog scores were employed as a covariate. When each CSF measure of pathology was included in the logistic regression, anosognosia no longer significantly predicted clinical progression $(p=0.09)$. For the participants with amnestic MCI included in the present analysis, the overall progression rate to probable $\mathrm{AD}$ in 2 years was $18.5 \%$. In the intact awareness group, progression to dementia was associated with a significant decline in awareness of illness from baseline (mean 22.27, SD 16.54) to 24-month follow-up (mean -4, SD $33.27, p=0.0006)$.

\section{Discussion}

The present study investigated the relationship among amyloid, tau, neurodegeneration, and anosognosia in amnestic 
MCI. The results demonstrate that impaired awareness of illness in $\mathrm{MCI}$ is accompanied by an increase in $\mathrm{AD}$ pathophysiology as well as decreased metabolism in neural structures vulnerable in $\mathrm{AD}$, independently of sex, education, age, and $A P O E \& 4$ status. The presence of anosognosia at baseline predicted hypometabolism and clinical progression at 24month follow-up independently of disease severity. Together, these findings highlight the importance of anosognosia as a clinical feature in amnestic MCI.

While MCIs in both groups did not differ on MMSE score, participants with MCI with impaired awareness of illness demonstrated lower global cognitive ability from the ADASCog. This divergence may be attributable to the ADAS-Cog being a more sensitive measure of cognitive dysfunction. ${ }^{13}$ Furthermore, the impaired awareness group performed worse on verbal memory and delayed recall, consistent with a recent meta-analysis of the neuropsychological correlates of impaired awareness in $\mathrm{MCI} .{ }^{14}$ Furthermore, because metacognitive monitoring abilities are also diminished in dementia, ${ }^{15}$ impaired awareness in MCI may be suggestive of incipient $\mathrm{AD}$.

Our findings of participants with MCI with impaired selfawareness having lower CSF $A \beta$, higher global $A \beta$ deposition, higher CSF p-tau, and higher CSF total tau supports a link between anosognosia and $\mathrm{AD}$ pathophysiology according to the $\mathrm{A} / \mathrm{T} / \mathrm{N}$ biomarker classification system. ${ }^{4}$ Specifically, in participants with MCI with impaired self-awareness, we found increased amyloid deposition in the PCC compared to individuals with intact self-awareness, a feature observed early in the $\mathrm{AD}$ process. ${ }^{16}$ Higher global amyloid burden has also been linked to decreased awareness of cognitive decline in MCI. ${ }^{17}$ Furthermore, results from a longitudinal study reported that individuals with impaired awareness of memory decline demonstrated increased tau pathology postmortem. ${ }^{18}$ While these results suggest that anosognosia in $\mathrm{MCI}$ is a clinical manifestation of $\mathrm{AD}$ pathophysiology, future work is needed to elucidate the role of cognitive impairment in anosognosia. Because relationships between lack of awareness of cognitive impairment and CSF amyloid and CSF p-tau were no longer significant when accounting for cognitive impairment, the regional distribution of neuropathology likely plays a role in the decline of illness awareness.

The association of anosognosia with decreased metabolism and increased amyloid load in the PCC, a key node of the default mode network (DMN), in MCI suggests a link between the PCC and the impairment of self-awareness. The $\mathrm{DMN}$ is a large-scale network of brain structures comprising the PCC/precuneus, medial temporal lobes, medial prefrontal cortex, as well as the lateral parietal cortex and is shown to be vulnerable in $\mathrm{AD}^{19}$ and $\mathrm{MCI}{ }^{20}$ Importantly, decreased metabolism in the PCC is regarded as a key feature of the early clinical stages of $\mathrm{AD} .^{21,22}$ While the pathologic features of $\mathrm{AD}$ typically first appear in the medial temporal lobes, ${ }^{23}$ previous work has suggested that the PCC is an area vulnerable to metabolic decline in early $\mathrm{AD}{ }^{16,24}$
Although the precise mechanism of decreased metabolism in the PCC leading to anosognosia in MCI is not known, multiple functional MRI studies have suggested that the PCC plays a key role in mental processing related to oneself ${ }^{25-27}$ and is involved in the retrieval of autobiographical memories. ${ }^{28}$ Furthermore, previous research has shown that less neuronal activity in the PCC is observed when patients with MCI with impaired awareness of illness engaged in a selfreferential mental task compared to patients with MCI with intact awareness. ${ }^{29}$ It is possible that patients' decreased mental activity relating to themselves may result in patients failing to integrate information about their recent functional difficulties and cognitive decline into their knowledge about themselves.

Anosognosia in amnestic MCI predicted hypometabolism in the PCC, bilateral medial temporal lobes, right lateral temporal lobe, and basal forebrain at 2-year follow-up. $\mathrm{AD}$ and $\mathrm{MCI}$ are characterized by significant loss of cholinergic neurons in the basal forebrain. ${ }^{30,31}$ Our results are also in line with previous results demonstrating that patients with $\mathrm{AD}$ with anosognosia had decreased glucose metabolism in the $\mathrm{PCC}^{32}$ and medial temporal lobes. ${ }^{33}$ Crucially, the PCC and medial and lateral temporal lobes are DMN structures particularly vulnerable in $\mathrm{AD}$, suggesting that the presence of anosognosia in MCI may be an important marker of incipient $\mathrm{AD}$ dementia. Correspondingly, individuals with amnestic MCI with impaired awareness of cognitive decline were 2.86 times more likely to progress to $\mathrm{AD}$ within a 2-year time frame than those with intact awareness. Our results are in agreement with previous research demonstrating that lack of awareness of cognitive decline typically appears 2-3 years before the onset of dementia. ${ }^{18}$

Awareness of cognitive decline is not incorporated into screening procedures such as the Alzheimer's Association recommendations for operationalizing the detection of cognitive impairment. ${ }^{34}$ If replicated, these findings demonstrating an increase in $\mathrm{AD}$ pathophysiology indicate that awareness assessments are a reasonable method to clinically screen individuals for interventions targeting $\mathrm{AD}$ pathophysiology in MCI. Indeed, anosognosia could provide clinicians with insights regarding clinical progression to dementia, though future work is needed to determine the methodology to most accurately assess awareness of illness.

While multiple procedures exist for assessing the extent of lack of awareness of cognitive decline, ${ }^{1}$ there is currently no ubiquitously accepted gold standard. Currently, approaches to quantifying lack of awareness of cognitive deficits fall into 3 main categories: (1) assessing the difference between subjective ability and objective score on a neuropsychological task, (2) assessment by a clinician, and (3) the discrepancy between patient and caregiver ratings of cognitive impairment. Because structured interviews with informants are already well-established in current clinical care (such as the Clinical Dementia Rating ${ }^{35}$ and the Informant Questionnaire 
on Cognitive Decline in the Elderly $[\mathrm{IQCODE}]^{36}$ ), clinicians may be able to gain important prognostic information by assessing a patient's awareness of illness with an informant.

Some methodologic considerations make replication of these findings highly desirable. The first is our operational measure of anosognosia; ECog is a measure of activities of daily living and not a measure of self-awareness in the strict sense. Another limitation of ECog is that it requires patients to recall their memories of past cognitive functioning, a faculty impaired in the anosognosia group. Despite the novelty of using the discrepancy of the ECog scores, the methodology of subtracting patient from caregiver scores has wide empirical support and is commonly employed in investigations of anosognosia across the $\mathrm{AD}$ spectrum., ${ }^{2,32,33}$ Furthermore, awareness of illness is not a binary phenomenon and future work is needed to establish the most appropriate method of determining the degree of awareness of illness across the $\mathrm{AD}$ spectrum. Another limitation of this research is that ADNI participants are self-selected, and thus may have been interested in participating due to having noticed a decline their cognitive abilities. Therefore, replication in another population-based cohort will strengthen confidence in these findings. However, a unique advantage of the ADNI cohort is that participants do not need to express a concern about their cognitive decline in order to be enrolled in the cohort, thus permitting the study of anosognosia for cognitive decline in MCI.

The results of the present study demonstrated that anosognosia in amnestic MCI is related to an increase in neuropathologic features of $\mathrm{AD}$ and increased risk of clinical progression to dementia. Overall, our findings highlight the importance of including assessments of self-awareness in medical diagnostic settings in order to provide additional insights into disease severity and clinical management across the $\mathrm{AD}$ spectrum.

\section{Author contributions}

J. Therriault, Dr. Ng, Dr. Pascoal: study concept, design, analysis and interpretation of data, creation of figures and manuscript draft. S. Mathotaarachchi: image data processing, analysis and interpretation of data, manuscript draft. M.S. Kang, H. Struyfs, M. Shin, A.L. Benedet: image data processing, analysis and interpretation of data, manuscript draft. I. Walpola: study concept and design, manuscript draft. Dr. Nair, Dr. Gauthier: study supervision and critical review of manuscript for intellectual content. Dr. Rosa-Neto: study concept, design, study supervision, critical review of manuscript for intellectual content.

\section{Study funding}

This work was supported by the Canadian Institutes of Health Research (MOP-11-51-31), the Alan Tiffin Foundation, the Alzheimer's Association (NIRG-12-92090, NIRP-12259245), the Fonds de Recherche du Québec-Santé, and the Centre for Studies on Prevention of $\mathrm{AD}$ (StoP-AD
Centre). Data collection and sharing for this project was funded by the ADNI (NIH grant U01 AG024904) and DOD ADNI (Department of Defence award number W81XWH12-2-0012). ADNI is funded by the National Institute on Aging, the National Institute of Biomedical Imaging and Bioengineering, and through contributions from the following: AbbVie; Alzheimer's Association; Alzheimer's Drug Discovery Foundation; Araclon Biotech; BioClinica, Inc.; Biogen; Bristol-Myers Squibb; CereSpir, Inc.; Eisai Inc.; Elan Pharmaceuticals, Inc.; Eli Lilly and Company; EuroImmun; F. Hoffmann-La Roche Ltd. and its affiliated company Genentech, Inc.; Fujirebio; GE Healthcare; IXICO Ltd.; Janssen Alzheimer Immunotherapy Research \& Development, LLC; Johnson \& Johnson Pharmaceutical Research \& Development LLC; Lumosity; Lundbeck; Merck \& Co., Inc.; Meso Scale Diagnostics, LLC; NeuroRx Research; Neurotrack Technologies; Novartis Pharmaceuticals Corporation; Pfizer Inc.; Piramal Imaging; Servier; Takeda Pharmaceutical Company; and Transition Therapeutics. The Canadian Institutes of Health Research also provides funds to support ADNI clinical sites in Canada. Private sector contributions are facilitated by the Foundation for the NIH (fnih.org). The grantee organization is the Northern California Institute for Research and Education, and the study is coordinated by the $\mathrm{AD}$ Cooperative Study at the University of California, San Diego. ADNI data were disseminated by the Laboratory for NeuroImaging at the University of Southern California.

\section{Disclosure}

J. Therriault, K.P. Ng, T.A. Pascoal, S. Mathotaarachchi, M.S. Kang, H. Struyfs, M. Shin, A.L. Benedet, I. Walpola, and V. Nair report no disclosures relevant to the manuscript. S. Gauthier received honoraria for serving on the scientific advisory boards of Alzheon, Axovant, Lilly, Lundbeck, Novartis, Schwabe, and TauRx and on the Data Safety Monitoring Board of a study sponsored by Eisai and studies run by the Alzheimer's Disease Cooperative Study and by the Alzheimer's Therapeutic Research Institute. P. Rosa-Neto reports no disclosures relevant to the manuscript. Go to Neurology.org/ $\mathrm{N}$ for full disclosures.

Received August 7, 2017. Accepted in final form December 5, 2017.

\section{References}

1. Starkstein SE. Anosognosia in Alzheimer's disease: diagnosis, frequency, mechanism and clinical correlates. Cortex 2014;61:64-73.

2. Starkstein S, Jorge R, Mizrahi R, Robinson RG. A diagnostic formulation for anosognosia in Alzheimer's disease. J Neurol Neurosurg Psychiatry 2006;77:719-725.

3. Gauthier S, Reisberg B, Zaudig M, et al. Mild cognitive impairment. Lancet 2006;367: $1262-1270$.

4. Jack CR, Bennet DA, Blennow $\mathrm{K}$, et al. A new classification system for $\mathrm{AD}$, independent of cognition $\mathrm{A} / \mathrm{T} / \mathrm{N}$ : an unbiased descriptive classification scheme for Alzheimer disease biomarkers. Neurology 2016;1-10.

5. Farias ST, Mungas D, Reed BR, et al. The measurement of everyday cognition: scale development and psychometric properties. Neuropsychology 2008;22:531-544.

6. Cacchione PZ, Powlishta KK, Grant EA, Buckles VD, Morris JC. Accuracy of collateral source reports in very mild to mild dementia of the Alzheimer type. J Am Geriatr Soc 2003;51:819-823.

7. Jorm AF. Assessment of cognitive impairment and dementia using informant reports. Clin Psychol Rev 1996;16:51-73.

8. Tierney MC, Szalai JP, Snow WG, Fisher RH. The prediction of Alzheimer disease: the role of patient and informant perceptions of cognitive deficits. Arch Neurol 1996; $53: 423-427$. 
9. Pascoal TA, Mathotaarachchi S, Mohades S, et al. Amyloid- $\beta$ and hyperphosphorylated tau synergy drives metabolic decline in preclinical Alzheimer's disease. Mol Psychiatry 2016;23:1-6.

10. Worsley KJ, Taylor JE, Tomaiuolo F, Lerch J. Unified univariate and multivariate random field theory. Neuroimage 2004;23(suppl 1):S189-S195.

11. Ng KP, Shin M, Gauthier S. Neuropsychiatric symptoms predict hypometabolism in preclinical Alzheimer disease. Neurology 2017;88:1814-1821.

12. Gräsbeck R. The evolution of the reference value concept. Clin Chem Lab Med 2004; 42:692-697.

13. Benge JF, Balsis S, Geraci L, Massman PJ, Doody RS. How well do the ADAS-cog and its subscales measure cognitive dysfunction in Alzheimer's disease? Demen Geriatr Cogn Disord 2009;28:63-69.

14. Piras F, Piras F, Orfei MD, Caltagirone C, Spalletta G. Self-awareness in mild cognitive impairment: quantitative evidence from systematic review and meta-analysis. Neurosci Biobehav Rev 2016;61:90-107.

15. Spalletta G, Piras F, Piras F, et al. Neuroanatomical correlates of awareness of illness in patients with amnestic mild cognitive impairment who will or will not convert to Alzheimer's disease. Cortex 2014;61.

16. Buckner RL. Molecular, structural, and functional characterization of Alzheimer's disease: evidence for a relationship between default activity, amyloid, and memory. J Neurosci 2005;25:7709-7717.

17. Vannini P, Amariglio R, Hanseeuw B, et al. Memory self-awareness in the preclinical and prodromal stages of Alzheimer's disease. Neuropsychologia 2017; 99:343-349.

18. Wilson RS, Boyle PA, Yu L, et al. Temporal course and pathologic basis of unawareness of memory loss in dementia. Neurology 2015;85:984-991.

19. Buckner RL, Andrews-Hanna JR, Schacter DL. The brain's default network: anatomy, function, and relevance to disease. Ann NY Acad Sci 2008;1124:1-38.

20. Mevel K, Chetelat G, Eustache F, Desgranges B. The default mode network in healthy aging and Alzheimer's disease. Int J Alzheimers Dis 2011;2011:535816.

21. Pengas G, Hodges JR, Watson P, Nestor PJ. Focal posterior cingulate atrophy in incipient Alzheimer's disease. Neurobiol Aging 2010;31:25-33.

22. Nestor PJ, Fryer TD, Smielewski P, Hodges JR. Limbic hypometabolism in Alzheimer's disease and mild cognitive impairment. Ann Neurol 2003;54:343-351.

23. Braak H, Braak E. Neuropathological stageing of Alzheimer-related changes. Acta Neuropathol 1991;82:239-259.
24. Minoshima S, Giordani B, Berent S, Frey KA, Foster NL, Kuhl DE. Metabolic reduction in the posterior cingulate cortex in very early Alzheimer's disease. Ann Neurol 1997;42:85-94.

25. Davey CG, Pujol J, Harrison BJ. Mapping the self in the brain's default mode network. Neuroimage 2016;132:390-397.

26. Andrews-Hanna JR, Reidler JS, Sepulcre J, Poulin R, Buckner RL. Functionalanatomic fractionation of the brain's default network. Neuron 2010;65:550-562.

27. Northoff G, Heinzel A, de Greck M, Bermpohl F, Dobrowolny H, Panksepp J. Selfreferential processing in our brain: a meta-analysis of imaging studies on the self. Neuroimage 2006;31:440-457.

28. Addis DR, Wong AT, Schacter DL. Remembering the past and imagining the future: common and distinct neural substrates during event construction and elaboration. Neuropsychologia 2007;45:1363-1377.

29. Ries ML, Jabbar BM, Schmitz TW, et al. Anosognosia in mild cognitive impairment: relationship to activation of cortical midline structures involved in self-appraisal. J Int Neuropsychol Soc 2007; 13:450-461.

30. Whitehouse PJ, Price DL, Struble RG, Clark AW, Coyle JT, Delon MR. Alzheimer's disease and senile dementia: loss of neurons in the basal forebrain. Science 1982;215: 1237-1239.

31. Mufson EJ, Ma SY, Dills J, et al. Loss of basal forebrain P75(NTR) immunoreactivity in subjects with mild cognitive impairment and Alzheimer's disease. J Comp Neurol 2002;443:136-153.

32. Perrotin A, Desgranges B, Landeau B, et al. Anosognosia in Alzheimer disease: disconnection between memory and self-related brain networks. Ann Neurol 2015;78: $477-486$.

33. Salmon E, Perani D, Herholz K, et al. Neural correlates of anosognosia for cognitive impairment in Alzheimer's disease. Hum Brain Mapp 2006;27:588-597.

34. Cordell CB, Borson S, Boustani M, et al. Alzheimer's Association recommendations for operationalizing the detection of cognitive impairment during the Medicare Annual Wellness Visit in a primary care setting. Alzheimers Demen 2013;9:141-150.

35. Morris JC. The Clinical Dementia Rating (CDR): current version and scoring rules. Neurology 1993;43:2412-2414.

36. Cherbuin N, Francis Jorm A. The Informant Questionnaire on Cognitive Decline in the Elderly (IQCODE). In: Abou-Saleh MT, Katona C, Kumar A, et al, editors. Principles and Practice of Geriatric Psychiatry, 3rd ed. Chichester, United Kingdom: John Wiley \& Sons, Ltd.; 2010:147-151. 


\section{Anosognosia predicts default mode network hypometabolism and clinical progression to dementia}

Joseph Therriault, BA, Kok Pin Ng, MRCP, Tharick A. Pascoal, MD, Sulantha Mathotaarachchi, MSc, Min Su Kang, BSc, Hanne Struyfs, MSc, Monica Shin, MSc, Andrea L. Benedet, MSc, Ishan Walpola, MSc, Vasavan Nair, MD, Serge Gauthier, MD, and Pedro Rosa-Neto, MD, PhD, For the Alzheimer's Disease Neuroimaging Initiative

Cite as: Neurology ${ }^{\circledR}$ 2018;90:e932-e939. doi:10.1212/WNL.0000000000005120

\author{
Correspondence \\ Dr. Rosa-Neto \\ pedro.rosa@mcgill.ca
}

\section{Study question}

What are the pathophysiologic mechanisms and clinical significance of anosognosia for cognitive decline in mild cognitive impairment (MCI)?

\section{Summary answer}

Anosognosia is linked to Alzheimer disease ( $\mathrm{AD}$ ) pathophysiology in vulnerable structures and predicts hypometabolism in the default mode network alongside an increased risk of progression to dementia.

\section{What is known and what this paper adds}

Anosognosia is commonly observed in $\mathrm{AD}$, but its meaning in $\mathrm{MCI}$ is poorly understood. This study provides evidence that anosognosia is associated with $\mathrm{AD}$ pathophysiology in patients with $\mathrm{MCI}$ and predicts disease progression.

\section{Participants and setting}

This study retrospectively examined 468 patients with amnestic MCI who participated in the Alzheimer's Disease Neuroimaging Initiative (ADNI), which was launched in 2003.

\section{Design, size, and duration}

The participants were classified into intact and impaired awareness groups based on discrepancies between patient and informant scores on the Everyday Cognition questionnaire. Voxel-based linear regression was used to evaluate how awareness status was related to amyloid- $\beta$ load, which was measured with ${ }^{18} \mathrm{~F}$-florbetapir at baseline, and regional brain glucose metabolism, which was measured with ${ }^{18} \mathrm{~F}$-fluorodeoxyglucose at baseline and after 24 months. Multivariate logistic regression was used to evaluate how awareness status was related to dementia progression, which was assessed with a battery of cognitive tests administered at baseline and after 24 months.

\section{Main results and the role of chance}

Of the 468 participants, 175 (37\%) had impaired awareness, and 293 (63\%) had intact awareness. Impaired status was associated with greater baseline amyloid- $\beta$ loads in the posterior cingulate cortex and glucose hypometabolism in areas representing the default mode network over 24 months. The frequency of progression to $\mathrm{AD}$ dementia was greater for the impaired awareness group ( $28 \%$ vs $12 \%$; odds ratio, 2.86 ; $95 \% \mathrm{CI}, 1.6-5.25)$.

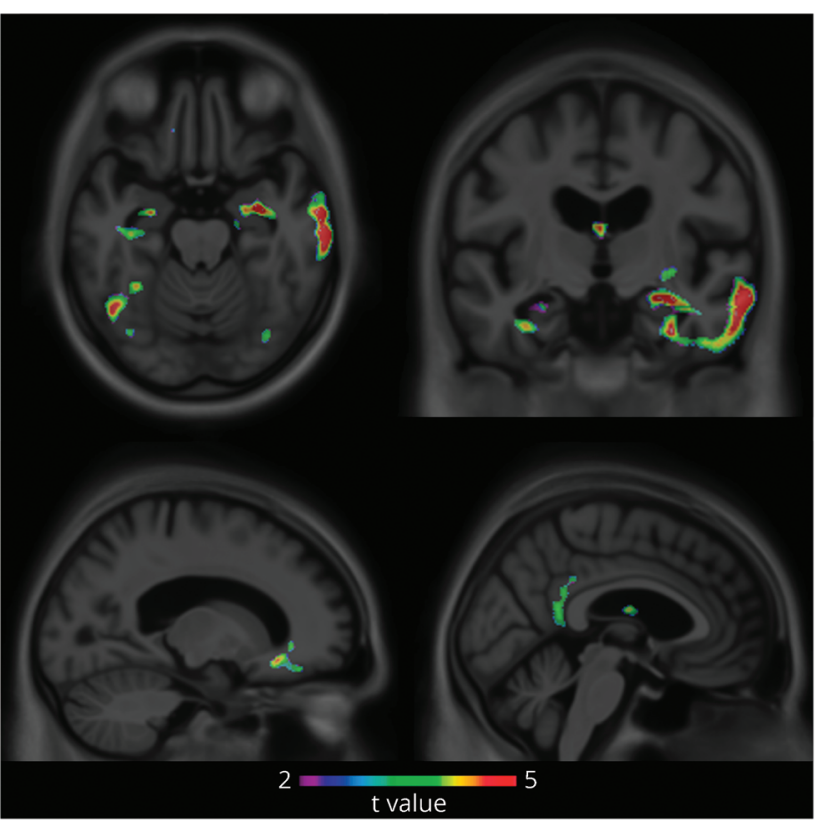

Bias, confounding, and other reasons for caution The Everyday Cognition questionnaire is an indirect measure of anosognosia. Furthermore, anosognosia occurs across a spectrum, not as a binary phenomenon.

\section{Generalizability to other populations}

The ADNI's subjects were self-selected, and so might have been interested in participating after noticing a decline in their cognitive abilities. This may limit generalizability to the general population of individuals with MCI.

\section{Study funding/potential competing interests}

This study was funded by Canadian government agencies and various medical research foundations. The ADNI was funded by the NIH and the US Department of Defense. Dr. Gauthier reports receiving honoraria for serving on advisory boards for various pharmaceutical companies and clinical trials related to $\mathrm{AD}$. Go to Neurology.org/ $\mathrm{N}$ for full disclosures. 


\section{Neurology}

\section{Anosognosia predicts default mode network hypometabolism and clinical progression to dementia}

Joseph Therriault, Kok Pin Ng, Tharick A. Pascoal, et al.

Neurology 2018;90;e932-e939 Published Online before print February 14, 2018

DOI 10.1212/WNL.0000000000005120

This information is current as of February 14, 2018

\section{Updated Information \&} Services

References

Citations

Subspecialty Collections

Permissions \& Licensing

Reprints including high resolution figures, can be found at: http://n.neurology.org/content/90/11/e932.full

This article cites 33 articles, 6 of which you can access for free at: http://n.neurology.org/content/90/11/e932.full\#ref-list-1

This article has been cited by 2 HighWire-hosted articles: http://n.neurology.org/content/90/11/e932.full\#\#otherarticles

This article, along with others on similar topics, appears in the following collection(s):

Alzheimer's disease

http://n.neurology.org/cgi/collection/alzheimers_disease

Assessment of cognitive disorders/dementia

http://n.neurology.org/cgi/collection/assessment_of_cognitive_disorder s_dementia

MCI (mild cognitive impairment)

http://n.neurology.org/cgi/collection/mci_mild_cognitive_impairment

PET

http://n.neurology.org/cgi/collection/pet

Information about reproducing this article in parts (figures,tables) or in its entirety can be found online at:

http://www.neurology.org/about/about_the_journal\#permissions

Information about ordering reprints can be found online:

http://n.neurology.org/subscribers/advertise

Neurology ${ }^{\circledR}$ is the official journal of the American Academy of Neurology. Published continuously since 1951, it is now a weekly with 48 issues per year. Copyright Copyright ( 2018 The Author(s). Published by Wolters Kluwer Health, Inc. on behalf of the American Academy of Neurology. All rights reserved. Print ISSN: 0028-3878. Online ISSN: 1526-632X.

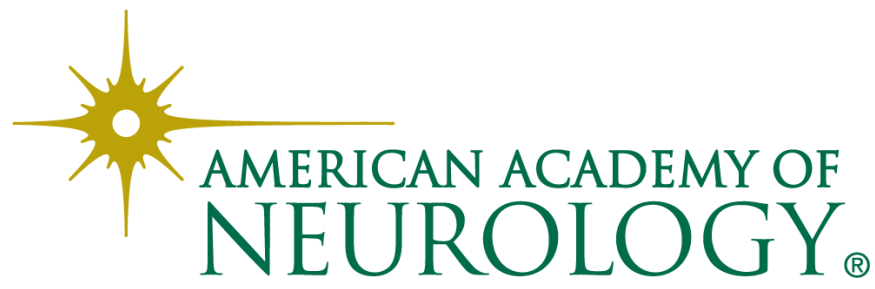

